\title{
Impact of Previous Abdominal Surgery on Laparoscopic Cystectomy/Oophorectomy Results: A Comparative Clinical Study
}

Hazim Wa, Roszaman $\mathrm{R}^{\mathrm{b}}$

aDept of Obstetrics \& Gynecology, Hospital Putrajaya

${ }^{b}$ Dept of Obstetrics \& Gynecology, International Islamic University of Malaysia

\section{ABSTRACT}

Introduction: In the past, patients with previous abdominal surgery were discouraged from laparoscopic surgery because of perceived increased risk of bowel injury caused by needle and trocar insertion. However, data on the feasibility and safety of surgery of this nature is increasing. We aim to evaluate the surgical outcome of laparoscopic ovarian cystectomy/oophorectomy in previous abdominal surgery. Methods: This is a cross-sectional study with descriptive analysis of retrospective data collection from the electronic medical records of women with laparoscopic ovarian cystectomy/ oophorectomy from January 2000 until Dec 2008. Results from patients with previous abdominal surgery were compared with those of women without prior abdominal surgery. Results: Three hundred and seventeen (317) laparoscopic cystectomies/ oophorectomies were performed during the study period. 71 patients $(22.5 \%)$ had previous history of abdominal surgery. Adhesions were found in $72 \%$ of patients versus $40 \%(p=0.001)$ who had previous abdominal surgery but the conversion to open surgery rate was similar $(3 \%, p<0.05)$. There was no significant difference in blood loss $(134.1 \mathrm{ml} \pm 18.6 \mathrm{vs} 119.0 \mathrm{ml} \pm 9.5)$, operating time $(107 \mathrm{~min} \pm 42.0$ versus $102.6 \mathrm{~min} \pm 42.6)$ and postoperative hospital stay ( 1.92 days \pm 1.0 vs 1.91 days \pm 0.7 ). The incidence of peri-operative and post-operative complication showed no significant difference in those who had undergone previous abdominal surgery than those without prior abdominal surgery $(\mathrm{p}=0.7)$. The overall complication rate in this series was $0.32 \%$. Conclusion: Laparoscopic cystectomy/ oophorectomy in the previous abdominal surgery is safe with no increase in morbidity.

KEYWORDS: Ovary, oophorectomy, surgery, laparoscopy, treatment outcome

\section{INTRODUCTION}

Operative laparoscopy is gaining popularity and widespread application in gynaecology. Nowadays, laparoscopic management of adnexal masses is the most frequently performed gynecology laparoscopic intervention. Laparoscopy in comparison to laparotomy has the advantages of lower morbidity, shorter length of hospital stay, decreased post-operative pain, lesser adhesion formation, better cosmetic results, faster recovery and reduced 0 verall cost of care. ${ }^{1}$

In the past, patients with previous abdominal surgery were discouraged from undergoing laparoscopic surgery because of increased risk of bowel injury caused by needle and trocar insertion. Complications occur two times more frequently in

\section{Corresponding author:}

Dr Roszaman Ramli

Associate Professor

Dept of Obstetrics and Gynaecology, Kulliyyah of

Medicine,

International Islamic University of Malaysia,

25250 Kuantan

Tel: 095132797

Fax : 095163798

Email: drroszaman@yahoo.com patients with previous laparotomy in a study of long series. $^{2}$

The potential risk for injury of organs adherent to the abdominal wall during veress needle or trocar insertion as well as the necessity for adhesiolysis and its attendant complications are the two major specific problems constraining surgeons from performing laparoscopic cystectomy/ oophorectomy for patients with previous abdominal surgery.

As surgeons gained more experience with laparoscopic techniques and technically advanced instrumentations were made, this limitation is no longer a contraindication for laparoscopic surgery. ${ }^{3}$ Data on safety of this surgery is emerging. The overall complication rate resulting from blind trans umbilical access in recent series is in the region of $1 \% .{ }^{4}$

Proper planning, safety rules, alternative entry method $^{5}$ and meticulous surgical techniques can prevent complications while still allowing patients to experience the benefits of minimally invasive surgery.

The aim of this study is to evaluate the safety of laparoscopic cystectomy and oophorectomy in previous abdominal surgery. 


\section{MATERIALS AND METHODS}

The electronic medical records from Hospital Putrajaya Database of all women who underwent laparoscopic cystectomy/ oophorectomy between January 2001 to December 2008 were reviewed. All patients with pre-operative diagnosis of benign adnexal mass who had undergone laparoscopic approaches where intraoperative finding was confirmed were included.

We studied the surgical outcome of laparoscopic cystectomy/ oophorectomy using veress needle insertion in patients with and without previous history of abdominal surgery.

All patients were operated under general anaesthesia with endotracheal intubation. Patients were placed at a Lloyd Davies position and a metal urinary catheter was inserted to empty the bladder. A cannula was placed into the uterine cavity if needed for uterine manipulation.

Umbilical veress needle insertion was the entry site of choice in the absence of previous abdominal surgery or in the presence of transverse suprapubic scar. Supraumbilical (at a point $2 \mathrm{~cm}$ above the proximal part of the scar in the epigastric region) or palmar point $(2-3 \mathrm{~cm}$ inferior to the middle of the last rib) was preferred for midline scar.

Aspiration was done with a syringe through the veress needle, which should not yield any blood, faeces or gas. This was followed by infusion of $10 \mathrm{cc}$ sterile water, which would not be able to be aspirated back. This, together with confirmatory "hanging drop test" (where the water level should have dropped on the veress needle upon entry into the peritoneal cavity) were performed in all cases.

Inflation was aimed to reach a pressure of 14-16 $\mathrm{mmHg}$. The $5 \mathrm{~mm}$ laparoscope was inserted through the umbilicus, and anxillary $5 \mathrm{~mm}$ trocars were inserted under direct vision. Placements of these trocars were decided according to the presence of adhesions as well as the size and location of the ovarian cyst.

Systematic inspection of the entire abdomen, including ovarian cyst, contralateral ovary, peritoneal cavity, paracolic gutter, liver surface, undersurface of the diaphragm, bowel surface and omentum were conducted.

Preventive technique was taken to avoid spillage and to clean up the pelvis after surgery if it did occur. Irrigation with copious amounts of fluid was done to remove particles of cyst contents, especially in the case of ruptured teratoma.

Post-operative analgesia was established by voltaren suppository +/- pethidine. Antibiotic prophylaxis was given in all cases. Patient's age, parity, ethnicity, number and type of prior abdominal surgeries, surgical technique, and operative findings were reviewed. Operative time, estimated blood loss, presence of adhesion, conversion to laparotomy and post-operative hospital stay were recorded. Complications of surgery and histopathology reports were also evaluated. A statistical analysis was performed using chi-square test or Fisher's exact test when appropriate. A p value of 0.05 was considered significant.

\section{RESULTS}

A total of 317 patients underwent laparoscopic cystectomy and oophorectomy during the study period. 71 patients $(22.5 \%)$ had previous abdominal surgery. Patients characteristics are listed in Table 1 . The median age was 29.8 years $( \pm 6.5)$; all patients were of pre-menopausal age. There were predominantly Malays (90.5\%) subjects, followed by Chinese and Indian, 3.5\% and 3.8\% respectively. 168 patients $(53.2 \%)$ were nulliparous.

71 patients $(22.5 \%)$ had previous abdominal surgery. The median number of prior abdominal surgery was 1 (range 1-4). 50 patients (70.4\%) had one previous abdominal surgery, 14 (19.7\%) had two previous surgeries and $6(8.5 \%)$ had three abdominal surgeries. Only one patient (1.4\%) had a history of four abdominal surgeries.

The great majority of peritoneal entry was via umbilical $(78.5 \%)$. Palmar entry and open method were chosen in $19.3 \%$ and $2.2 \%$ respectively.

The types of abdominal surgery were: lower transverse Lower Segment Caesarean Section (LSCS) 20 (28.2\%), open cystectomy/ oophorectomy $13(18.3 \%)$, appendicectomy $7(10 \%)$, mixed LSCS and open cystectomy/ oophorectomy 8 (11\%), laparoscopic \& dye insufflations 4 , and laparoscopic cystectomy 19.

Adhesions were found in $72 \%$ of patients versus $40 \%(\mathrm{p}=0.001)$ who had previous abdominal surgery. However, patients with previous abdominal surgery had a similar open conversion rate $(3 \%, p$ $<0.05$ ). In cases with previous surgery, conversion to open laparotomy was mostly due to the dense adhesion. There were 11 cases, which required conversion to open surgery (3.5\%).

The mean duration of surgery was 82.9 minutes (45-170 $\mathrm{min})$ and average blood loss was 170.5 cc. Four out of 71 patients $(5.6 \%)$ had an estimated blood loss greater than $500 \mathrm{ml}$. Three patients $(2.8 \%)$ had surgery time more than three hours.

There was no significant difference in the mean blood loss (134.1 ml \pm 18.6 vs $119.0 \pm 9.5)$, operating time $(107 \mathrm{~min} \pm 42.0$ vs $102.6 \pm 42.6)$ and post-operative stay (1.92 days \pm 1.0 vs $1.91 \pm$ 0.7 ) in those who had undergone previous abdominal 
surgery than those without prior abdominal surgery. The overall complication rate was $0.32 \%$. The incidence of peri-operative and post-operative complication showed no significant difference $(p=0.7)$. There was no bowel or vascular injury. Two cases had wound breakdown where none of them required re-surgery. No deaths occurred in these study subjects.

The mean duration of hospital stay was 1.9 days. Only 1 patient $(1.4 \%)$ had a length of hospital stay more than three days.

\section{DISCUSSION}

Previous abdominal surgery has been recognized as one of the most important risk factors for laparoscopic surgery. But the question is how the morbidity can be kept at the minimum while maintaining its superior benefit over open surgery.

Laparoscopic access is the crucial most part of the surgery where veress needle was blindly inserted to create pneumoperitoneum followed by primary trocar. Obtaining access to the peritoneal cavity in laparoscopic surgery is more difficult in patients with previous abdominopelvic surgery, as it can become a difficult, time-consuming, and occasionally hazardous procedure. The majority of complications (30-50\%), occur during surgical access, ${ }^{6,7}$ with vascular and bowel injuries the most serious sequele. ${ }^{8}$

Formation and extent of adhesions are unpredictable. The incidence of abdominal wall adhesions was significantly more frequent after prior laparotomy. Midline laparotomy above the level of the umbilicus was found to be associated with the highest rate of adhesion (67\%). ${ }^{9}$ The risk of adhesion for suprapubic transverse incision was $28 \% .^{10}$ Adhesions, when present involved mainly omentum (96\%) and bowel (29\%). However, from the data available we are not able to determine the rate of adhesion from the different types of scar in this study.

Bowel injuries due to the veress needle are rare when safety steps and rules are observed strictly. ${ }^{11}$ However, the safest approach, confirmatory tests and access technique, in these cases remain uncertain. ${ }^{12}$

There was no major complication such as visceral or vessel's injury in this series. The overall complication rate in this study was $0.32 \%$ and they were mainly port site infection. The incidence of peri-operative and post-operative complication was not significantly different $(p=0.7)$ whether or not patient had previous abdominal surgery. A higher post-operative complication rate of $13.4 \%$ was reported in a recent study of similar nature. ${ }^{13}$ Left upper quadrant or palmar point varess needle insertion was our preferred site for peritoneal entry when midline scar was present. This area is generally free of adhesions and we would consider using in patients with suspected periumbilical adhesions or after 2-3 failed insufflation attempts at the umbilicus. ${ }^{14}$ The risk of either by the varess needle or the trocar cannula is minimized with adequate skin traction and utilization of 'hanging drop test' we routinely performed.

Open laparoscopy approach was used only in a limited number of cases $(2.2 \%)$ in our unit. ${ }^{15}$ We used it as a last resort, when there is a difficult umbilical or palmar entry. Open laparoscopy is not shown to reduce the incidence of visceral injury, ${ }^{16}$ but is recommended in patients with suspected adhesion, obese or very thin. The use of an optical trocar is less favoured by many and does not confer a significantadvantageoverthetraditional Hassonentry. ${ }^{17}$

Conversion to laparotomy occurred in 11 cases (3.5\%) and was mostly due to dense adhesion. Timely judgment has to be made regarding when to convert; balancing between reducing complication rate against an increase in operating time and cost. ${ }^{18}$ This conversion rate is comparable with the rate of $4.6 \%$ in a study conducted at an organized laparoscopic training centre. ${ }^{19}$ We employ sharp dissection and stitching as opposed to coagulation techniques for tissue dissection in the presence of adhesion. This follows the recommendation ${ }^{2}$ for a safe surgical practice to reduce the incidence of bowel injury.

The average number of previous operations was 1.4 per person with the maximum number of four previous surgeries. There was no increase in major complication rate even in a patient with previous multiple abdominal surgery in this series. As shown in a large prospective series for mainly advanced laparoscopic surgery, prior abdominal surgery has no significant effect on the overall morbidity rate. ${ }^{20}$

Inadvertent enterotomy in laparoscopic abdominal surgery is especially dangerous if unrecognized during primary operation. ${ }^{21}$ Complications, if occurred must be recognized and repaired immediately. Any patient with signs of peritonitis or increased abdominal pain after laparoscopic surgery must promptly be investigated. The spillage of cyst contents in laparoscopic management of dermoid cysts can potentially lead to complications such as chemical peritonitis. In our series, spillage rate was encountered in five patients $(1.6 \%)$ but there was no case with evidence of clinical chemical peritonitis. Copious irrigation of the pelvis was performed in all cases with the cysts removed via endobag retrieval technique.

What are the odds that the cyst is malignant? Benign pathology was identified in all cases. The most common diagnosis was endometriosis (44\%), mature teratoma $(31 \%)$, corpus luteum cyst $(10 \%)$, simple cyst $(1 \%)$ and others $(14 \%)$. There was no case of malignancy found in our series. A detailed pre-operative assessment as well as thorough and systematic intra-operative evaluation is indeed crucial. 
Table 1. Patient characteristics

\begin{tabular}{ll}
\hline Demographic data & Mean (SD) \\
\hline Age & $29.9(6.5)$ \\
Parity & $2.7 \quad(24.8)$ \\
\hline
\end{tabular}

Table 2: Previous surgery VS Number of previous surgery: Surgical outcome

\section{Previous abdominal Surgery No previous surgery $\quad$ value}

\begin{tabular}{llll}
\hline $\begin{array}{l}\text { Adhesion (\%) } \\
\text { Conversion Rate (\%) }\end{array}$ & $72 \%$ & $40 \%$ & $0.001^{*}$ \\
& $3 \%$ & $3 \%$ & NS \\
Blood Loss (ml) & $134.1 \pm 18.6$ & $119.0 \pm 9.5$ & NS \\
$\begin{array}{lll}\text { Operating Time (min) } \\
\text { Hospital Stay (days) } \\
\text { Complication (n) }\end{array}$ & $107 \pm 42$ & $102.6 \pm 42.6$ & NS \\
& $1.92 \pm 1.0$ & $1.91 \pm 0.7$ & NS \\
\hline
\end{tabular}

Since the introduction of operative laparoscopic technique in our department, the laparoscopic approach has been limited to adnexal masses thought to be benign pre-operatively. Selection of cases, careful individualized risk assessment and observing safety rule on peritoneal entry technique contributed considerably to the safety and successful outcome.

\section{CONCLUSION}

The data in this series shows that laparoscopic cystectomy/oophorectomy for benign ovarian cyst in previous abdominal surgery is safe and associated with low morbidity.

\section{REFERENCES}

1. Medeiros LR, Stein AT, Fachel J, Garry R, Furness S. Laparoscopy versus laparotomy for benign ovarian tumor: a systematic review and meta-analysis. Int J Gynecol Cancer 2008;18:387-99.

2. Kolmorgen K. Laparoscopy complications in previously operated patients. Zentralbl Gynakol 1998;120:191-4.

3. Purnichescu V, Cheret-Benoist A, Von Theobald $\mathrm{P}$, et al. Laparoscopic management of pelvic mass in pregnancy. J Gynecol Obstet Biol Reprod 2006;35: 388-95.

4. Lecuru F, Leonard F, Jais JP, et al. Laparos copy in patients with prior surgery: results of the blind approach. JSLS 2001;5:13-6.

5. Rafii A, Camatte $S$, Lelièvre L, Daraï $E$,
Lécuru F. Previous abdominal surgery and closed entry for gynaecological laparoscopy: a prospective study. BJOG 2005; 112:100-2.

6. Tarik A, Fehmi C. Complications of gynaecological laparoscopy--a retrospective analysis of 3572 cases from a single institute. J Obstet Gynaecol 2004;24:813-6.

7. Mac Cordick C, Lecuru F, Rizk E, et al. Morbidity of laparoscopy for gynecological surgery. Results of a prospective monocentre study. Surg Endosc 1999;13:57-61.

8. Chapron C, Pierre F, Lacroix S, Querleu D, Lansac J, Dubuisson JB. Major vascular injuries during gynaecologic laparoscopy. J Am Coll Surg 1997;185:461-5.

9. Brill Al, Nezhat F, Nezhat CH, Nezhat C. The incidence of adhesions after prior laparotomy: a laparoscopic appraisal. Obstet Gynecol 1995;85:269-72.

10. Bieber EJ, Barnes RB. Anterior abdominal wall adhesions after laparotomy or laparoscopy. J Am Assoc Gynecol Laparosc 1997;4:353-6.

11. Bonjer HJ, Hazebroec EJ, Kazemier G, et al. Open versus closed establishment of pneumoperitoneum in laparoscopic surgery. Br J Surg 1997;84:599-602.

12. Rosen D, Lam A, Chapman M, Carlton M, Cario G. Methods of creating pneumoperitoneum: a review of techniques and complications. Obstet Gynecol Surv 1998;53:167-74.

13. Kumakiri J, Kikuchi I, Kitade M, et al. Incidence of complications during gynecologic laparoscopic surgery in patients after previous laparotomy. J Minim 
Invasive Gynecol 2010;17:480-6.

14. Vilos GA, Ternamian A, Dempster J, Laberge PY. Laparoscopic entry: a review of techniques, technologies, and complications. J Obstet Gynaecol Can 2007; 29:433-65.

15. Hasson HM. Open laparoscopy: report of 150 cases. J Reprod Med 1974;12:234238.

16. Jansen FW, Kolkman W, Bakkum EA, et al. Complications of laparoscopy: an inquiry about closed- versus open-entry technique. Am J Obstet Gynecol 2004; 190:634-8.

17. Tinelli A, Malvasi A, Istre 0 , et al. Abdominal access in gynaecological laparoscopy: a comparison between direct optical and blind closed access by Verres needle. Eur J Obstet Gynecol Reprod Biol 2010;148:191-4.

18. Binenbaum SJ, Goldfarb MA. Inadvertent Enterotomy in Minimally Invasive Abdominal Surgery. $J$ of the Society of Laparoendoscopic Surgeons 2006;10: 336-40.

19. Minig L, Velazco A, Lamm M, et al. Evaluation of laparoscopic management of gynecologic emergencies by residents. Int J Gynaecol Obstet 2010; 111:62-7.

20. Leonard F, Lecuru F, Rizk E, et al. Perioperative morbidity of gynecological laparoscopy. A prospective monocenter observational study. Acta Obstet Gynecol Scand 2000;79:129-34.

21. Van der Voort M. Bowel injury as a complication of laparoscopy. Br J Surg 2004;91(10):1253-8. 